\title{
SÍNTESE DE NANOTUBOS DE CARBONO DE PAREDE SIMPLES POR SUBLIMAÇÃo DE GRAFITE EM ATMOSFERA DE HÉLIO
}

\author{
José Gino Venegas Romero, Carlos Alberto Luengo, John G Huber* e José Maurício Rosolen\#
}

Departamento de Física Aplicada, Instituto de Física "Gleb Wataghin”, UNICAMP, 13083-970 Campinas - SP

Recebido em 23/2/01; aceito em 20/6/01

\begin{abstract}
SYNTHESIS OF SINGLE-WALL NANOTUBES BY PYROLYSIS OF GRAPHITE IN HELIUM ATMOSPHERE. Macroscopic samples of fullerene nanostructures are obtained in a modified arc furnace using the electric arc method with a Helium atmosphere at low pressures. High purity graphite rods are used as electrodes but, when drilled and the orifices filled with powders of transition metals $(\mathrm{Fe}, \mathrm{Co}, \mathrm{Ni})$ acting as catalysts, the resulting particles are carbon nanostructures of the fullerene family, known as Single Wall Nanotubes (SWNTs). They have typical diameters of $1.4 \mathrm{~nm}$, lengths up to tenths of microns and they are arranged together in bundles containing several SWNTs. Those samples are observed and analyzed using Scanning Electron Microscopy (SEM) and Transmission Electron Microscopy (TEM) techniques.
\end{abstract}

Keywords: nanotubes; bundles; arc-furnace.

\section{INTRODUÇÃo}

Os nanotubos de carbono descobertos em 1991 por S. Iijima ${ }^{1}$ são de dois tipos: os nanotubos de carbono de parede simples (NCPS) e de parede múltipla (NCPM). Os NCPS são formados de uma folha de grafite enrolada com extremos fechados por metades de fulerenos, e os NCPM são formados por vários NCPS concêntricos como em um cabo coaxial.

A maioria das propriedades dos NCPS depende do seu diâmetro e ângulo "chiral", também chamado helicidade. Estes dois parâmetros diâmetro e helicidade, resultam completamente definidos pelos dois índices de Hamada $(n, m)^{2}$. Como é mostrado na Figura 1A, um NCPS pode ser construído por uma folha de grafite enrolada de tal forma, que coincidam dois sítios cristalograficamente equivalentes de sua rede hexagonal. $\mathrm{O}$ vetor $\mathbf{C}$ chamado chiral, que define a posição relativa dos dois sítios é definido mediante dois números inteiros $(\mathrm{n}, \mathrm{m})$ e pelos vetores unitários da rede hexagonal $\mathbf{a}_{\mathbf{1}}$ e $\mathbf{a}_{\mathbf{2}}\left(\mathbf{C}=\mathrm{n} \mathbf{a}_{\mathbf{1}}+\right.$ $\mathrm{ma}_{2}$ ). Um tubo é chamado "armchair" se $\mathrm{n}=\mathrm{m}$, e "zigzag" quando $\mathrm{m}=0$. Em todos os outros casos $(\mathrm{n} \neq \mathrm{m} \neq 0)$ os tubos são chamados "chiral" e estão definidos pelo ângulo de helicidade $\phi$. Se $\phi$ varia de $0^{\circ}$ a $30^{\circ}$ podemos gerar tubos partindo do tipo "armchair" até o "zigzag" como limites, passando pelos tubos "chiral". Na Figura 1B, são mostrados os três tipos de tubos mencionados.

Os nanotubos de carbono, especialmente os NCPS, têm propriedades eletrônicas excepcionais. Em termos dos índices (n,m), um nanotubo é metálico quando n-m é múltiplo de 3 , em caso contrário é semicondutor ${ }^{4}$. Todos os nanotubos armchair são metálicos, enquanto que os zigzag e chiral podem ser metálicos ou semicondutores. Os avanços recentes em nanotecnologia têm permitido a construção de uma nova classe de nanodispositivos: um diodo de NCPS, uma junção de dois feixes superpostos de NCPS, e transistores de elétron simples e de efeito de campo a partir de um NCPS ${ }^{5,6}$. Quando é aplicado um campo elétrico intenso da ordem de $10^{7} \mathrm{~V} / \mathrm{cm}$ sobre a superfície de um sólido com potencial elétrico negativo, são emiti-

* Pesquisador independente, 501 N. Boulevard, \#7, Richmond, VA 23220, USA

\# Departamento de Química, FFCLRP, USP, 14040-901 Ribeirão Preto - SP dos elétrons pelo efeito de tunelamento. Este fenômeno é conhecido como emissão de elétrons de campo. Um campo de tão alta intensidade pode ser obtido na ponta de um feixe de NCPS uma vez que os campos elétricos se concentram nas pontas. Baseando-se neste fenômeno, foram construídos emissores de elétrons de campo para microscópios de emissão de campo a partir de feixes de NCPS ${ }^{7}$.

O espaço dentro dos NCPS dá a possibilidade de armazenar grandes quantidades de átomos ou moléculas, em particular, hidrogênio. Assim, seriam desenvolvidos novos materiais de armazenamento com elevada capacidade, leves, e alta estabilidade, os quais, poderiam ser aplicados para mover veículos com celas combustíveis ${ }^{8}$. Os cálculos mostram que os átomos de hidrogênio podem ser adsorvidos tanto nas partes interna e externa dos NCPS, como no seu espaço interno. A espectroscopia Raman revelou a existência de moléculas de hidrogênio no espaço interno de NCPS, fato confirmado por simulações de dinâmica molecular. A capacidade de armazenamento aumenta linearmente com o diâmetro dos NCPS 9 .

É conhecido que os NCPS formam feixes com uma rede triangular empacotada bidimensionalmente ${ }^{10}$. A microscopia eletrônica de transmissão ${ }^{11,12} \mathrm{e}$ as caracterizações de difração de raios $\mathrm{X}^{13}$, mostram que os sítios intersticiais entre os NCPS, podem ser ocupados por intercalantes tendo assim, aplicação como materiais para eletrodos de baterias secundárias. Os NCPS produzidos sem purificar, mostraram uma composição de saturação reversível de $\mathrm{Li}_{1.2} \mathrm{C}_{6}\left(450 \mathrm{mAh} \cdot \mathrm{g}^{-1}\right)$. Depois de remover as fases de impurezas mediante filtração, a composição de saturação incrementou-se para $\mathrm{Li}_{1.6} \mathrm{C}_{6}\left(600 \mathrm{mAh} . \mathrm{g}^{-1}\right)$ atingindo inclusive a composição de $\mathrm{Li}_{2.7} \mathrm{C}_{6}\left(1000 \mathrm{mAh} \cdot \mathrm{g}^{-1}\right)^{14}$. É conhecido que a resistência à tensão das fibras aumenta, quando elas são cada vez mais grafitizadas ao longo de seu eixo. Portanto, os NCPS seriam as fibras mais resistentes na natureza. Efetivamente, foram realizadas medidas do módulo de Young de NCPS com diâmetros entre 1.0 e 1.5 $\mathrm{nm}$ resultando com um valor médio do módulo de Young de $1.25 \mathrm{TPa}^{15}$, aproximadamente 1000 vezes maior que do aço.

\section{SÍNTESE}

Existem vários métodos de produção de NCPS a partir de carbonos como grafite, sendo os principais os seguintes: descarga por arco elétrico ${ }^{16}$ e ablação por laser ${ }^{17}$, ambos geralmente numa atmosfera 

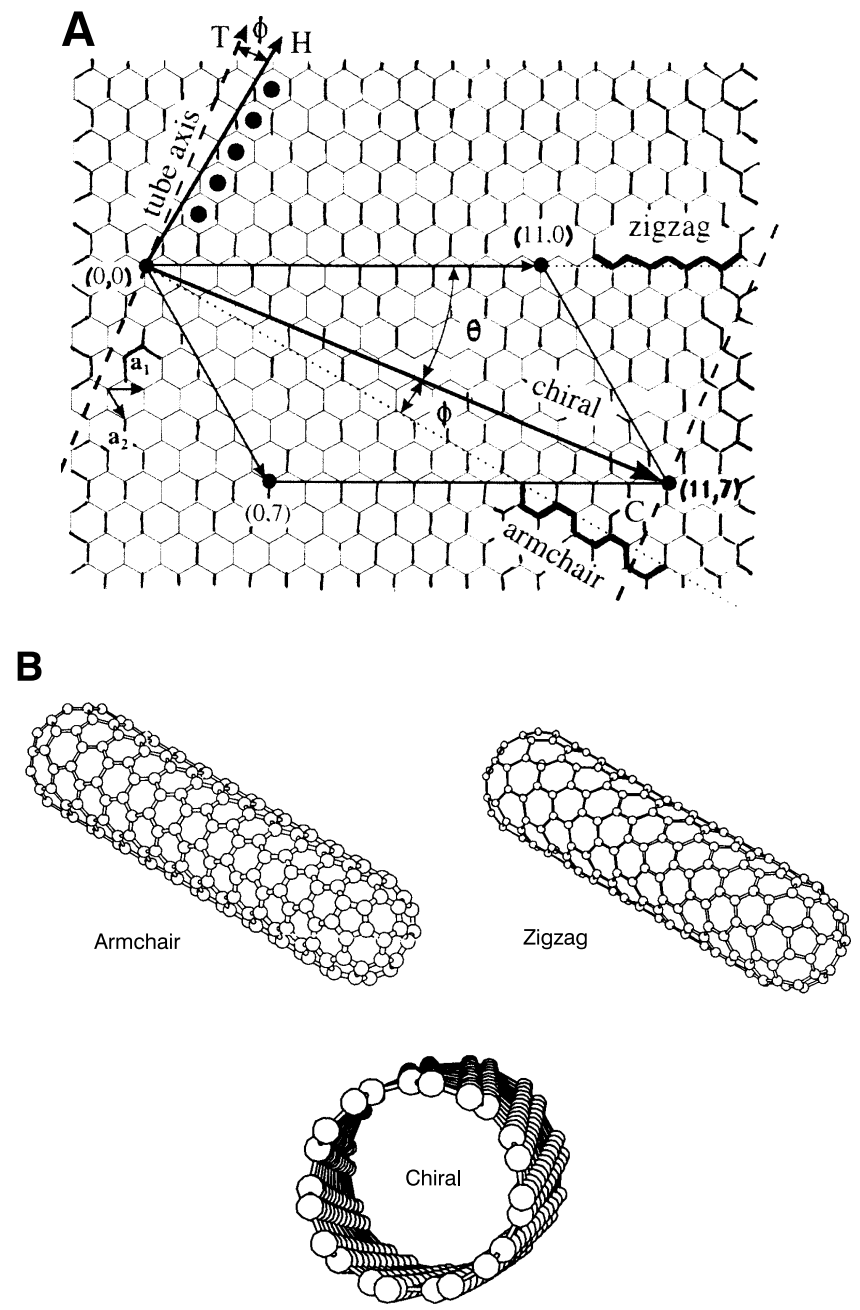

Figura 1. Configuração geométrica dos NCPS ${ }^{3}$. A- A construção de um nanotubo enrolando uma folha de grafite na direção do vetor chiral $\boldsymbol{C}$ de tal modo que a origem $(0,0)$ coincida com seu extremo $(11,7)$. Se o enrolamento fosse na direção das linhas pontilhadas, teríamos tubos armchair e zigzag. Todos os outros tubos chiral, são gerados pelo ângulo de helicidade $\phi$ medido a partir da direção armchair, ou pelo ângulo $\theta$ medido a partir da direção zigzag $\left(\phi=30^{\circ}-\theta\right)$. As linhas tracejadas são perpendiculares à $\boldsymbol{C}$ e indicam a direção do eixo do tubo definida pelo vetor $\boldsymbol{T}$. $O$ vetor $\boldsymbol{H}$ é perpendicular à direção armchair, indicando a direção dos hexágonos mais próximos marcados com pontos grandes. $O$ ângulo entre $\boldsymbol{T}$ e $\boldsymbol{H}$ também é $\phi$. B- Nanotubos zigzag, armchair, e chiral.

de hélio a baixa pressão, para atingir melhores rendimentos de produção. No primeiro método o princípio de funcionamento está baseado numa descarga elétrica gerada entre dois eletrodos de grafite, porém, um deles (ânodo) está preenchido com partículas metálicas catalisadoras de metais de transição (principalmente $\mathrm{Fe}, \mathrm{Ni}, \mathrm{Co}$ e suas combinações). A alta temperatura entre os eletrodos permite a sublimação do carbono formando-se os NCPS. No segundo método, o carbono é vaporizado a partir da superfície de um disco de grafite misturado também com metais de transição, utilizando um laser pulsado e focalizado. $\mathrm{O}$ alvo de grafite é inserido no meio de um tubo de quartzo mantido numa temperatura controlada da ordem de $1200^{\circ} \mathrm{C}$. Os NCPS formados são arrastados mediante um fluxo de hélio e posteriormente condensados numa superfície coletora refrigerada de cobre.
No nosso laboratório, foram produzidos feixes de NCPS num reator baseado no arco de descarga numa atmosfera de hélio a baixa pressão, como descrito detalhadamente na referência 18. Foi aplicada uma corrente de 120 A (D.C.) e uma pressão base de 400 Torr de hélio. Em dois experimentos diferentes foram utilizados como catalisadores os compostos $\mathrm{Zr}\left(\mathrm{Fe}_{0.5} \mathrm{Ni}_{0.5}\right)_{2}$ e $\mathrm{Zr}\left(\mathrm{Co}_{0.5} \mathrm{Ni}_{0.5}\right)_{2}$ obtidos também no nosso laboratório e preenchidos em varetas de grafite ultrapuro (UF-4S da Carbon of America) na proporção em massa de 1:4. Em volta do cátodo é formado um colar frágil com aspecto de flor, altamente denso em NCPS ${ }^{18}$.

\section{CARACTERIZAÇÃO}

O material coletado do cátodo e obtido a partir do composto $\mathrm{Zr}\left(\mathrm{Fe}_{05} \mathrm{Ni}_{05}\right)_{2}$, foi observado e analisado mediante um microscópio eletrônico de varredura FESEM- JEOL, JSM-6340F e um microscópio eletrônico de transmissão ZISS CEM 902, com câmera CCD PROSCAN, 80kV.

Na Figura 2A, é mostrada uma imagem MEV do material obtido sem purificar. Observa-se a presença de nanopartículas de carbono (manchas menos claras) e nanopartículas do catalisador (manchas claras). Os NCPS formam feixes com impurezas de carbono em sua superfície ao longo de seu comprimento. Os feixes são curvos e altamente densos mostrando um alto rendimento de síntese de NCPS.

A imagem TEM da Figura 2B, foi obtida a partir do mesmo material purificado mediante ultrasom. Nesta imagem observa-se um feixe de NCPS formado por 8 NCPS com diâmetros de aproximadamente $1.4 \mathrm{~nm}$, valor confirmado pela análise de espectroscopia Raman $^{19}$, e varias dezenas de microns de comprimento. Na Figura 2B é mostrada uma região onde não se encontram as nanopartículas de catalisadores ou de grafite. O tratamento com ultra-som aplicado desagrega os feixes de nanotubos da fuligem, não remove as nanopartículas de catalisador ou grafites. A remoção destas nanopartículas exige tratamentos oxidativos específicos para cada tipo de catalisador utilizado na produção dos NCPS.

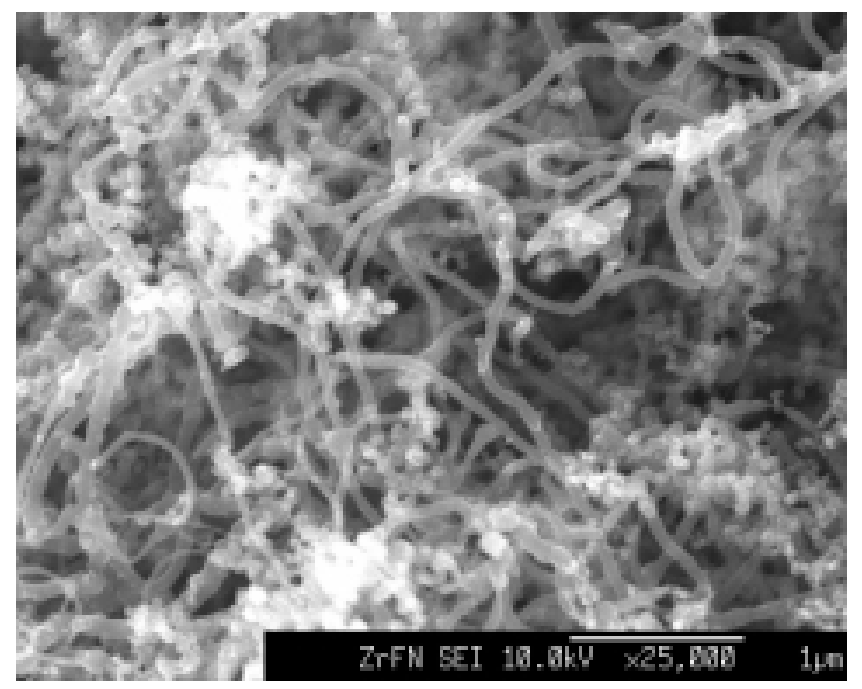

Figura 2A. STM do material de carbono coletado na região do reator chamada de colar (região de contorno do anodo do reator).

\section{CONCLUSÕES}

O reator utilizado permite produzir feixes de NCPS, sendo que cada feixe contém entre 6 e 8 nanotubos, com diâmetros da ordem de 1,4 nm tal como foi confirmado por espectroscopia Raman, e comprimentos que atingem várias dezenas de microns, a partir de 


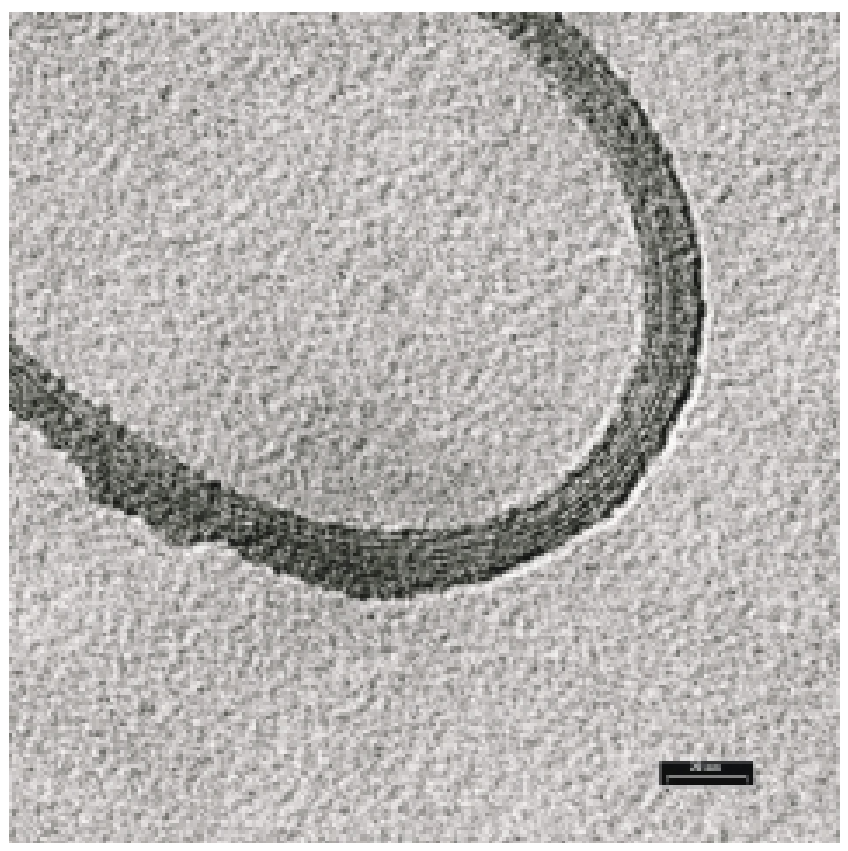

Figura 2B. STM de um feixe de nanotubos de parede simples presente no material obtido no reator depois de um tratamento de ultra-som para desagregação de feixes e separação de fuligem.

compostos de $\mathrm{Zr}\left(\mathrm{Fe}_{0.5} \mathrm{Ni}_{0.5}\right)_{2}$. Resultados similares foram também observados a partir do composto $\mathrm{Zr}\left(\mathrm{Co}_{0.5} \mathrm{Ni}_{0.5}\right)_{2}$.

Com o objetivo de esclarecer a influência do catalisador na formação dos feixes de NCPSs (comprimento, diâmetro) e na formação de nanopartículas (catalisadores e grafite), está sendo realizado atualmente uma série de experimentos com vários tipos de catalisadores tais como a $\mathrm{CeNi}_{2}$ e $\mathrm{ZrFe}_{2}$ e vários óxidos $(\mathrm{NiO}, \mathrm{CoO}$, etc).

\section{REFERÊNCIAS}

1. Iijima, S.; Nature (London) 1991, 354, 56.

2. Hamada, N.; Sawada, S.; Oshiyama, S.; Phys. Rev. Lett. 1992, 68, 1579.

3. Saito, R.; Fujita, M.; Dresselhaus, G.; Dresselhaus, M.S.; Phys. Rev. B 1992, 46, 1804.

4. Saito, R.; Fujita, M.; Dresselhaus, M.; Dresselhaus, G.; Appl. Phys. Lett. 1992, 60, 2204; Saito, R.; Fujita, M.; Dresselhaus, M.; Dresselhaus, G.; Phys. Rev. B 1992, 46, 1804.

5. Tans, S.; Verschueren, A.; Dekker, C.; Nature 1998, 393, 49.

6. Lefevbre, J.; Antonov, R.D.; Radosavljevic, M.; Lynch, J.F.; Llaguno, M.; Johnson, A.T.; Carbon 2000, 38, 1745.

7. Saito Y.; Uemura, S.; Carbon 2000, 38, 169.

8. Dillon, A. C.; Jones, K.M.; Bekkedahl, T.A.; Kiang, C.H.; Bethune, D.S.; Heben, M.J.; Nature 1997, 386, 377.

9. Lee, S.M.; Park, K.S.; Choi, Y.; Park, Y.S.; Bok, J.M.; Bae, D.J.; Nahm, K.S.; Choi Y.G.; Yu, S.C.; Kim, N.; Frauenheim, T.; Lee, Y.H.; Synth. Met. 2000, 113, 209.

10. Thess A.; Lee, R.; Nikdaev, P.; Dai, H.; Petit, P.; Robert, J.; Xu, C.; Lee, Y.H.; Kim, S.G.; Rinzler, A.G.; Colbert, D.T.; Scuseria, G.E.; Tomanek, D.; Fischer, J.E.; Smalley, R.E.; Science 1996, 273, 483.

11. Suzuki, S.; Bower, C.; Zhou, O.; Chem. Phys. Lett. 1998, 285, 230.

12. Grigorian, L.; Williams, K.A.; Fang, S.; Sumanasekera, G.U.; Loper, A.L.; Dickey, E.C.; Pennycook, S.J.; Eckland, P.C.; Phys. Rev. Lett. 1998, 80, 5560.

13. Bower, C.; Kleinhammes, A.; Wu, Y.; Zhou, O.; Chem. Phys. Lett. 1998, $288,481$.

14. Gao, B.; Kleinhammes, A.; Tang, X.P.; Bower, C.; Fleming, L.; Wu, Y.; Zhou, O.; Chem. Phys. Lett. 1999, 307, 153.

15. Krishnan, A.; Dujardin, E.; Ebbesen, T.W.; Yianilos, P.N.; Treacy, M.M.J.; Phys. Rev. B 1998, 58, 14013.

16. Krätschmer, W.; Lamb, L.D.; Fostiropoulos, K.; Huffman, D.R.; Nature (London) 1990, 347, 354.

17. Guo, T.; Nikolaev, P.; Rinzler, A.G.; Tomanek, D.; Colbert, D.T.; Smalley, R.E.; J. Phys. Chem. 1995, 99, 10694.

18. Huber, J.G.; Romero, J.V.; Spivey, J.D.; and Luengo, C.A.; Quim. Nova 2001, 24, 898 .

19. Corio, P.; Temperini, M.L.A.; Santos, P.S.; Romero, J.V.; Huber, J.G.; Luengo, C.A.; Chem. Phys. Lett., no prelo. 